\title{
15th International World Federation of Neurology Workshop on Huntington's Disease, 31 August-3 September 1993, Boston, Massachusetts, USA
}

This biennial meeting of the World Federation of Neurology Research Group on Huntington's Disease took place less than six months after the report from the HD Collaborative Research Group of the identification of an expanded and unstable CAG trinucleotide repeat in the gene IT 15 in subjects with HD. One hundred and seventy-one scientists and clinicians from 16 countries met at the same time as representatives of the International Huntington Association from 27 countries. Much of the data presented at the workshop resulted from work generated after the discovery of the HD gene and it is clear that the pace of research into the molecular basis for the disease phenotype has accelerated.

Dr J Gusella (Boston) gave the first presentation of the scientific workshop, outlining the contribution made by disequilibrium and haplotype studies to the fine localisation of IT15. The gene comprises 67 exons spread over $100 \mathrm{~kb}$ of genomic DNA and encodes mRNA transcripts of 13.5 and $10.5 \mathrm{~kb}$ which differ at the $3^{\prime}$ untranslated region. Both transcripts are expressed in all tissues but the ratio of the two varies. Interestingly, it appears that in fetal brain the $13.5 \mathrm{~kb}$ transcript predominates but in adult brain the reverse is true. Direct mutation analysis in his laboratory has shown that in 80 to $85 \%$ of cases where the disease is maternally transmitted there is a change in the repeat length, enlarging in two-thirds of these cases and diminishing in the remaining onethird with a change of between one and four repeats. There is also change in $85 \%$ of paternal transmissions and, of these, one-third show a more significant expansion (at most a doubling). As has been found by most groups, he reported that the age at onset/repeat length correlations are strongest for disease of early onset with the correlation dropping to approximately -0.4 for smaller repeats. The question of the origin of the expansion was raised and it has been shown that alleles with an intermediate' repeat length of 33 to 38 occur on the common (one-third of subjects) HD haplotype associated with the delta 2642 deletion codon (this loss of a glutamine does not appear to affect the function of the huntingtin protein as it is also found in $7 \%$ of the normal popula- tion). It is not clear whether these intermediate length repeats are themselves any more unstable than 'normal' length repeats or whether further expansion occurs because of an effect of the overall haplotype. Goldberg (Vancouver) presented data on the molecular analysis of 'new mutation' cases of HD showing that of 21 such cases, 18 were expansion positive. Examination of their parents showed that one carried an allele with 30 to 38 repeats (called an intermediate allele by other workers) and that expansion to a 'full mutation' only occurred during transmission through the male germline and this was associated with advanced paternal age. This finding has implications in counselling as it means that for sibs of an isolated case the probability of them passing on an expanded allele (disease associated allele) is lower for female than male sibs. Unfortunately, a probability value is not yet available and it is likely to require a meta analysis of the pooled data to derive this. Davis (London) showed that of subjects with a phenotype typical of HD but with a negative family history, $90 \%$ were mutation positive, as opposed to $50 \%$ of those with an atypical presentation. Several lines of work suggest that the repeat instability is prezygotic: variability of repeat length is seen in sperm, where it is typically larger than in blood cells; three sets of identical twins are concordant for repeat length (showing that instability occurs before the twinning event); and comparison of cell lines cultured for 7 to 10 years show no evidence for somatic mosaicism.

Dr M Hayden addressed the issues of sensitivity and specificity of the mutation analysis, reporting that of 1000 patients with a clinical diagnosis of $\mathrm{HD}$, only 33 cases had repeat lengths of less than 37 . Fifteen of these could be attributed to clerical/diagnostic or sampling error; of those remaining, four came from families where other affected subjects had the expansion, six were 'new mutations', and eight had family histories of a similar disorder and other affected subjects also had normal repeat lengths. Examination of the data presented in several posters showed that some groups have excluded such cases from their analyses; the Vancouver group reported only 360 of the above 1000 cases in their poster and gave a 
lower limit of 38 repeats in 'affected' subjects. Analyses of 1910 affected and 3585 normal chromosomes were presented from USA (Boston), Canada (Vancouver), UK (Manchester and Cardiff), Holland (Leiden), Germany (Bochum), and Italy (Rome). Repeat length ranged from 27 to 121 on HD and 7 to 37 on control chromosomes. Excluding the one affected subject from Manchester with 27 repeats and the Cardiff case with 30, the lower limit for the HD range rises to 36 . Cases defined as 'juvenile HD' showed a range from 44 to 121 repeats. At the other extreme, the group from Leuven (Belgium) reported, in HD families, subjects who have repeat lengths between 39 and 41 and the onset of chorea aged 70 , cognitive decline at the age of 80 but no chorea at 90 years, and neither chorea nor cognitive decline also at the age of 90 ! These cases emphasise that caution needs to be exercised when dealing with subjects with positive presymptomatic tests and the counsellor should resist requests to prognosticate on a possible age for symptom onset. One possible area for concern highlighted was that the repeat ranges reported so far make no allowance for the polymorphism in the polylysine repeat sequence which is also amplified by the primers reported initially. Dr Hayden (Canada) reported that $60 \%$ of subjects had seven copies of the repeat but up to $1 \%$ had 12 copies. Interpretation of a presymptomatic test result of 35 to $42 \mathrm{CAG}$ repeats would depend on the number of CCG repeats also amplified; a difference of five could change the assignment status from normal to abnormal in a number of situations.

Several groups presented data on the distribution of the mRNA in tissue sections from normal and affected brain using oligonucleotide probes. Kremer (Vancouver) showed that the mRNA in normal and HD brain contains the expanded CAG sequence. These workers and others hypothesise that the expansion has its effects at the protein level. There were limited data on the use of antibodies to localise the protein product. Only one of two abstracted posters was displayed; this showed antibody labelling of the nuclear region in neurones from HD brains but no staining in control tissue. The interpretation of these preliminary findings is unclear.

Outside the genetic aspects, evidence was presented that besides the familiar caudate hypometabolism seen on positron emission tomograph (PET) scanning, there is general hypometabolism in the resting HD brain that spares the cerebellum. Immunocytochemical studies on postmortem HD brains have previously suggested that striatal output GABA/ enkephalin neurones of the 'indirect' pathway to the lateral globus pallidus (presumed to bear largely $\mathrm{D}_{2}$ dopamine receptors on their cell bodies) are lost earlier and to a more severe degree than GABA/substance $P$ neurones of the 'direct' pathway to medial globus pallidus $\left(D_{1}\right)$. However, PET scans using " $\mathrm{C}$-raclopride and ${ }^{11} \mathrm{C}-\mathrm{SCH} 23390$ as ligands for $\mathrm{D}_{1}$ and $\mathrm{D}_{2}$ receptors respectively suggest a parallel 40 to $50 \%$ loss of both receptors, in both rigid and choreic subjects. The session on neurochemistry stressed that the absolute and relative degrees of loss of enkephalin immunoreactivity from the lateral pallidum and substantia nigra reticulata seemed to be the same whether one looks at the brains of the rigid versus choreic, or young versus old, subjects. New neurochemical studies examined the distribution of cannabinoid, 5HT1D, and substance $P$ receptor and glutamate receptor subunit binding, and concentrations of synaptophysin and protein kinase $c$ in normal persons, and their pattern of loss in HD basal ganglia.

There was much speculation on the search for effective treatment(s) for the disease. We are now leaving the rather unsatisfactory era of symptomatic treatment to concentrate on neuroprotective (slowing progression or delaying onset), preventive (halting progression or preventing onset), or genetic therapy. Chase (NIH) gave a rather gloomy picture of the results from pilot trials with putative neuroprotective agents such as baclofen (anti-glutaminergic) and tocopherol (anti-oxidant) and outlined newer agents such as antagonists of NMDA, AMPA, and glycine and calcium channel blockers.

Isaacson (Belmont) provided an overview of the potential for neural transplantation in HD, citing animal work showing that cells from the fetal lateral ganglionic process differentiated into striatal cells when transplanted and that only this cell type had a beneficial effect in a (excitotoxic) primate model. Additional postulated candidates for implantation include the use of genetically engineered cells producing growth factors or neurotransmitters or both and the possibility of masking major histocompatibility antigens to allow transgenic grafts. It was generally agreed that some cell source other than freshly aborted human tissue ultimately needs to be found, and that much more animal experimentation needs to be done, particularly when a transgenic model for HD becomes available.

Whatever therapeutic candidate one chooses, the methodology of clinical trials is going to be crucial, particularly as it may be critical to detect not just clinical improvement but also slowed progression. Longitudinal data for most complementary investigations in HD are scanty, but there is useful clinical follow up data from the Venezuelan pedigree, from the baclofen study, and from an additional group of 78 patients followed by Shoulson et al at Rochester, NY, for a mean of 3.4 years, who generated data presented at this meeting. Plans are well advanced for a collaborative multicentre longitudinal clinical study of the natural history of large cohorts of HD subjects both in the United States and in Europe as a necessary prelude to any study of any potentially disease modifying treatment.

The scientific sessions of the workshop ran, for the most part, concurrently with the meetings of the International Huntington Association. The joint session on the second day provided the opportunity for both sides to express their thoughts on some of the ethical 
dilemmas which have arisen with the availability of direct mutation analysis. Any clinician expecting a definitive answer to the question of whether a person at $25 \%$ risk should be permitted to obtain a predictive test result when the intervening parent at $50 \%$ risk declines testing would have been disappointed. It was the opinion of most members of the IHA that an adult at $25 \%$ risk did have the right to obtain such a result but that the spouse of a man at $50 \%$ risk did not have the right to obtain a prenatal result on an at risk $(25 \%)$ pregnancy. The final text of the revised guidelines on presymptomatic testing are eagerly awaited by many clinicians.

What of the future? At the previous meeting two years ago in Cardiff, the task of isolating the gene for Huntington's disease had yet to be completed. The struggle to understand how the expansion in IT15 affects the huntingtin protein and causes the clinical manifestations of the disease has only now begun. Perhaps in
Leuven in 1995 at the 16 th meeting we will be addressing the question: what are the options for treatment?

Scientists and clinicians with a continuing research commitment in HD and who have attended, or will attend, these meetings can become members of the WFN Research Group at the next meeting if they have a proposer and seconder from within the group, and send a brief CV to the Secretary General, Dr Michael Hayden at the University of British Columbia in Vancouver, Canada.

JOHN C MACMILLAN Institute of Medical Genetics, University of Wales College of Medicine, Cardiff CF4 $4 X N$, UK

NIALL P QUINN The National Hospital, Queen Square, 